\title{
FUTURE VERTEX DETECTOR \\ FOR OPEN CHARM MEASUREMENTS WITH THE NA61/SHINE EXPERIMENT AT THE CERN SPS*
}

\author{
Yasir Ali, Pawee Staszel \\ The Marian Smoluchowski Institute of Physics, Jagiellonian University \\ Reymonta 4, 30-059 Kraków, Poland
}

(Received July 29, 2013)

\begin{abstract}
A studies related to the future Vertex Detector (VD) for the $D^{0}$ meson (open charm) measurements in the NA61/SHINE experiment at the CERN SPS are presented. The VD will allow for precise track reconstruction at the target proximity. The measurement of $D^{0}$ mesons provide a unique opportunity to test the validity of perturbative QCD-based and statistical models of nucleus-nucleus collisions at SPS energies. Moreover, the production and propagation of charm in heavy-ion collisions is sensitive to the high-density effects and phase transitions. These measurements will be a challenging adventure due to the low multiplicity and short life time of $D^{0}$ mesons. This study also addresses the issue of VD optimization and the emphasis is put on the prospect of the development of a VD based on CMOS technology.
\end{abstract}

DOI:10.5506/APhysPolBSupp.6.1081

PACS numbers: 29.40.Wk

\section{Introduction}

NA61/SHINE [1] is a fixed target experiment at the CERN SPS. NA61 is a large acceptance hadron spectrometer whose main components are inherited from NA49 experiment. The setup shown in Fig. 1 includes the beam position detectors, Cherenkov counters, and the scintillator stations located upstream of the target. These detectors provide information of timing, type and position of beam particles, and are located upstream of the target. Four large volume Time Projection Chambers (TPCs) serve as tracking detectors, Time-Of-Flight (TOF) walls are mainly used for hadron identification, and finally, Projectile Spectator Detector (PSD), which is a large hadronic

* Presented at the Symposium on Applied Nuclear Physics and Innovative Technologies, Kraków, Poland, June 3-6, 2013. 
calorimeter positioned downstream of the TOF detectors. The main purpose of PSD is to measure the energy of projectile fragments and provide information on reaction centralities. In order to measure $D^{0}$ mesons the current experimental setup has to be upgraded with a dedicated VD. The top view of the NA61/SHINE experimental setup with the indicated position of the future VD is shown in Fig. 1.

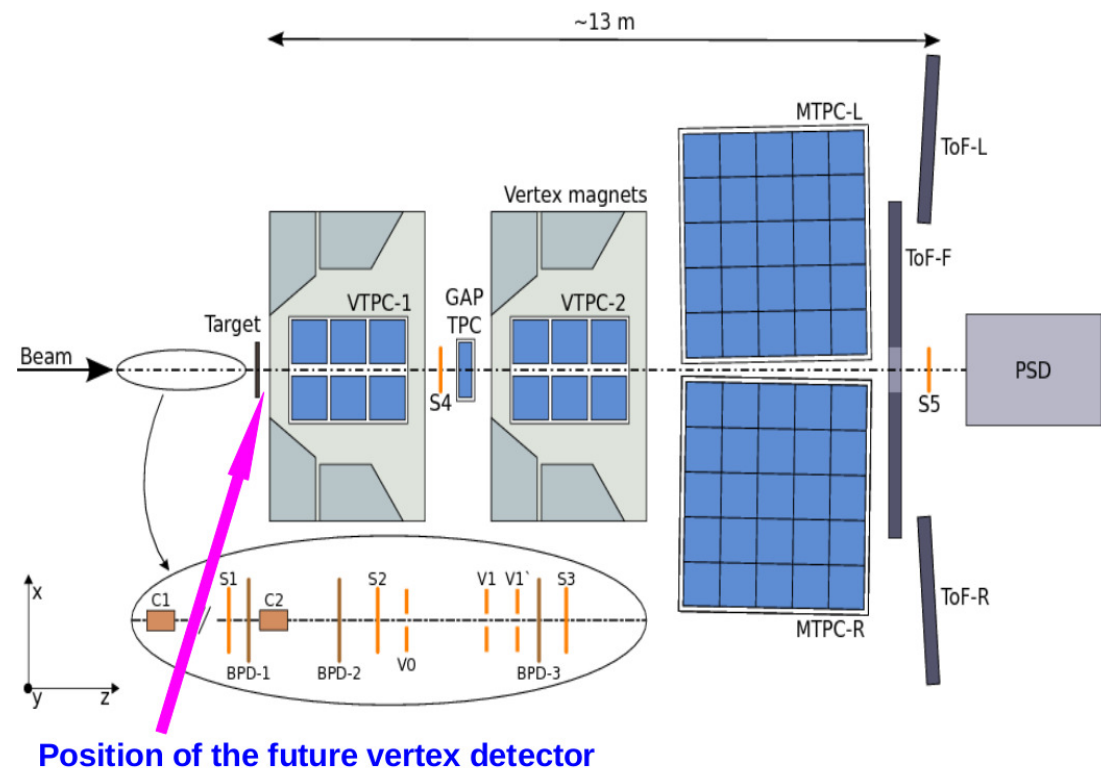

Fig. 1. The layout of the NA61/SHINE experimental setup (top view, not to scale) with mentioned position for the future VD.

\section{Vertex detector studies}

By using the developed simulation framework we were able to determine what kind of requirements have to be fulfilled by the anticipated detector. These requirements refer to radiation tolerance, position resolution, hit density, and readout speed. The MIMOSA-26 sensor whose architecture is based on the Monolithic Active Pixel Sensor (MAPS) with fast binary readout [2] have been chosen as a basic detection element of the VD stations. The selection of MIMOSA-26 was mostly driven by the very high hit occupancy in the stations found from simulations. The hit occupancy in the VDS1 generated by a single central $\mathrm{Pb}+\mathrm{Pb}$ collision at the top SPS energy of $158 \mathrm{~A} \mathrm{GeV}$ reaches value of about $5 \mathrm{hits} / \mathrm{mm}^{2} /$ event in the innermost part of the detector. Such a high occupancy cannot be handled by existing micropattern gas detectors mostly because of their one-dimensional readout. For 
this technology, even for the hardly achievable strip pitch size on the level of $100 \mu \mathrm{m}$, the two-particle separation for the indicated occupancy is not feasible. On the other hand, MIMOSA-26 offers high pixel granularity with a real 2-dimensional readout. It is expected that during the data taking period the VD will be exposed to high level of radiation. The sensor performance can be deteriorated by the non-ionizing energy loss (NIEL). To account for the NIEL effects two sources of particles have to be considered, namely, particles produced in hadronic nucleus-nucleus interaction and $\delta$-electrons. Based on the anticipated beam intensity and the interaction rates the particle fluxes through the VD detector have been calculated. Integrating the fluxes over 1 month (in 1-2 months NA61/SHINE can collect about $50 \mathrm{M}$ central $\mathrm{Pb}+\mathrm{Pb}$ events) and using the so-called displacement damage function [3] the fluencies of $2.6 \times 10^{10} \mathrm{n}_{\mathrm{eq}} / \mathrm{cm}^{2}$ and $4.2 \times 10^{10} \mathrm{n}_{\mathrm{eq}} / \mathrm{cm}^{2}$ in the most illuminated area of sensors were found for $\delta$-electrons and hadrons, respectively. The tests on proton beam of MIMOSA-26 sensors proved that these devices can handle fluencies up to $3 \times 10^{12} \mathrm{n}_{\mathrm{eq}} / \mathrm{cm}^{2}$ which provides large safety margin for using them in the NA61/SHINE application [4].
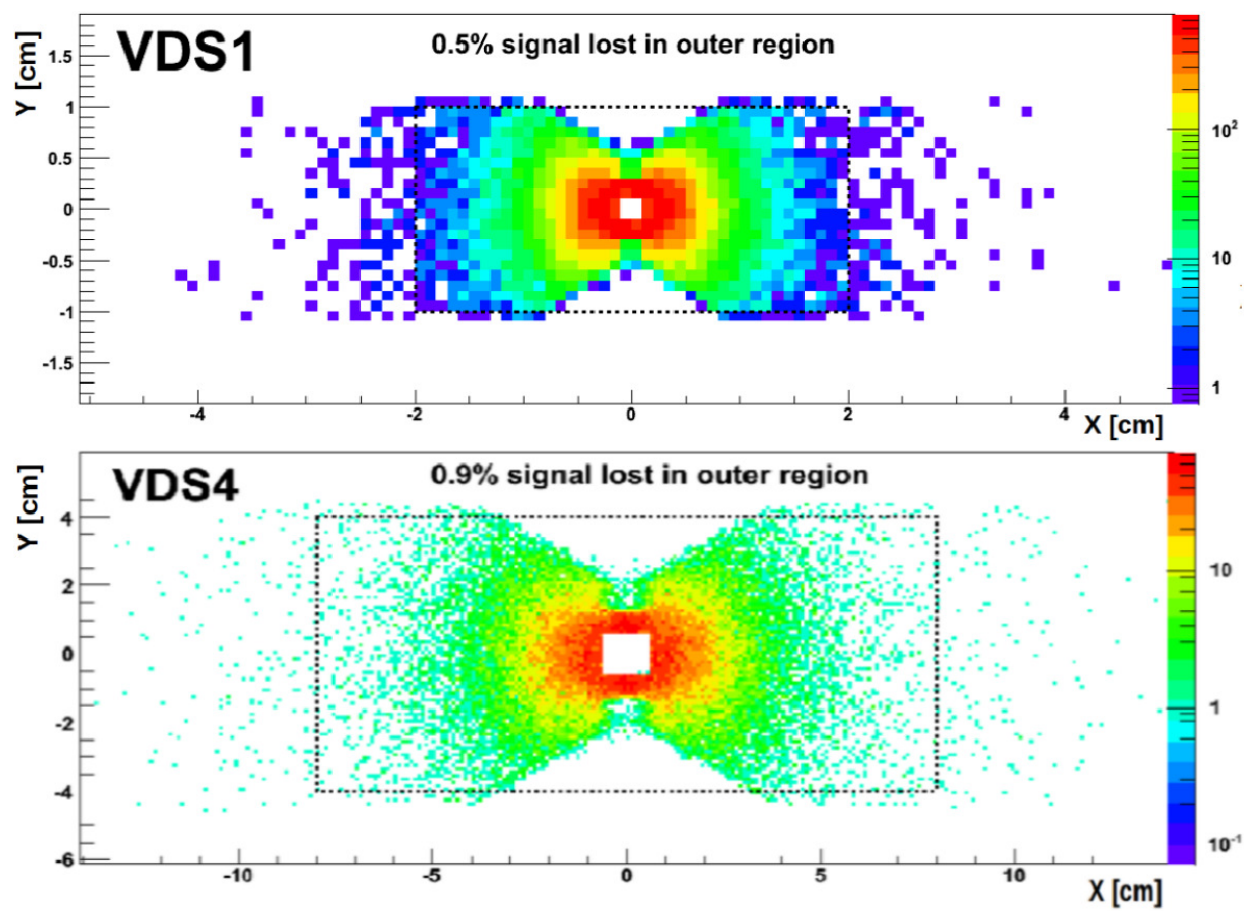

Fig. 2. Distribution of hits in VD stations VDS1 and VDS4. 
In addition, MIMOSA-26 sensors can be thinned to $50 \mu \mathrm{m}$ thickness which means that the sensor itself provides a small fraction of the total material budget as compared to the amount of material introduced by mechanical support, cooling fluid and readout cabling.

VD consists of four vertex detector stations, named as VDS1, VDS2, VDS3 and VDS4 located respectively at distances of $5 \mathrm{~cm}, 10 \mathrm{~cm}, 15 \mathrm{~cm}$, and $20 \mathrm{~cm}$ from the target. Figure 2 shows hits $(x, y)$ distribution generated by signal tracks in VDS1 and VDS4. We found that for VDS1 99.5\% of signal tracks are localized within the $2 \times 4 \mathrm{~cm}^{2}$ box. To cover the remaining $0.5 \%$ we would need to extend the box in the $x$ direction almost by the factor of 2 . For stations VDS2-VDS4 we just extend size of the boxes in proportion to their distance from the target. So we got dimensions of: $4 \times 8 \mathrm{~cm}^{2}, 6 \times 12 \mathrm{~cm}^{2}$ and $8 \times 16 \mathrm{~cm}^{2}$ for VDS2, VDS3 and VDS4, respectively. These dimensions are shown for VDS1 and VDS4 as a dashed boxes in Fig. 2.

\section{Conclusion}

The $D^{0}$ meson measurements are feasible in the NA61/SHINE experiment with a proposed VD based on MIMOSA-26 sensors. These sensors fulfil all requirements imposed by the NA61/SHINE application and will be used as a basic detection element in the VD stations. These devices provide high readout speed and also high position resolution required to reconstruct the $D^{0}$ decay vertices. They are also sufficiently radiation hard to be used in the foreseen measurement of open charm in the NA61/SHINE experiment.

We acknowledge the support by the Foundation for Polish Science MPD program, co-financed by the European Union within the European Regional Development Fund and the National Science Centre of Poland (grant UMO-2012/04/M/ST2/00816).

\section{REFERENCES}

[1] The NA61/SHINE homepage http://na61.web.cern.ch

[2] MIMOSA26 User Manual, Institut Pluridisciplinaire Hubert Curien IN2P3-CNRS.

[3] A. Vasilescu, ROSE Internal Note ROSE/TN/97-2 (1997).

[4] M. Winter, CBM Prograss Report, GSI/FAIR (2010). 